\title{
Critical Behavior of Phase Interfaces in Porous Media: Analysis of Scaling Properties with the Use of Noncoherent and Coherent Light
}

\author{
D. A. Zimnyakov ${ }^{a, b}, *$, A. V. Sadovoi ${ }^{a}$, M. A. Vilenskii ${ }^{a}$, P. V. Zakharov ${ }^{c}$, and R. Myllylä ${ }^{d}$ \\ a Saratov State University, Moskovskaya ul. 155, Saratov, 410012 Russia \\ ${ }^{b}$ Institute of Problems of Fine Mechanics and Control, Russian Academy of Sciences, Saratov, 410028 Russia \\ ${ }^{c}$ University of Fribourg, 1700, Fribourg, Switzerland \\ ${ }^{d}$ University of Oulu, FI-90014, Oulu, Finland \\ *e-mail: zimnykov@sgu.ru
}

\begin{abstract}
Image sequences of the surface of disordered layers of porous medium (paper) obtained under noncoherent and coherent illumination during capillary rise of a liquid are analyzed. As a result, principles that govern the critical behavior of the interface between liquid and gaseous phases during its pinning are established. By a cumulant analysis of speckle-modulated images of the surface and by the statistical analysis of binarized difference images of the surface under noncoherent illumination, it is shown that the macroscopic dynamics of the interface at the stage of pinning is mainly controlled by the power law dependence of the appearance rate of local instabilities (avalanches) of the interface on the critical parameter, whereas the growth dynamics of the local instabilities is controlled by the diffusion of a liquid in a layer and weakly depends on the critical parameter. A phenomenological model is proposed for the macroscopic dynamics of the phase interface for interpreting experimental data. The values of critical indices are determined that characterize the samples under test within this model. These values are compared with the results of numerical simulation for discrete models of directed percolation corresponding to the Kardar-Parisi-Zhang equation.
\end{abstract}

PACS numbers: 68.35.Rh, 68.35.Ct, 68.35.Fx, 68.35.Ja

\section{INTRODUCTION}

The critical behavior of various nonideal systems with weak structural order is one of the fundamental phenomena in the physics of condensed state and statistical physics, which have been intensively studied for several decades. For example, this behavior is typical of charge-density waves [1], domain walls in ferromagnetic materials [2], and contact lines at the interface between liquid and solid phases [3].

A typical manifestation of critical behavior is the growth of an interface between liquid and gaseous phases in disordered porous media under nonstationary mass transfer with small values of the average velocity $\bar{V}$ on a macroscopic level (on a spatial scale on the order of the characteristic size of the medium in a direction perpendicular to the velocity of the interface) [4$6]$. When the resulting force $F_{\Sigma}$ that controls the motion of a phase interface in a porous medium is close to a certain threshold value $F_{c}$, which depends on the structural characteristics of the medium and the parameters of interaction between the liquid phase and the medium, the function $\bar{V}\left(F_{\Sigma}\right)$ exhibits scaling behavior: $\bar{V} \propto\left(F_{\Sigma} / F_{c}-1\right)^{\theta}$, which is indicative of the critical behavior of the system [7]. When the critical parameter $f=F_{\Sigma} / F_{c}-1$ tends to zero, the phase interface is pinned. At the pinning stage, the evolution of the interface represents a series of acts of appearance, growth, and decay of local instabilities - avalanches-whose characteristic size and rate of appearance are also described by power law functions of the critical parameter $f$.

Most of the papers on investigation into the critical behavior of a phase interface in porous media published in recent decades are mainly devoted to the structural properties of growing interfaces by numerical simulation using various discrete models of multiphase porous systems [8-11]. Different authors reported controversial data on the scaling properties of the phase interface, and the experimental verification of such models was mainly performed by analyzing the structural characteristics of the interface on the macroscopic level (for example, by analyzing the mean width, height, and fractal dimension of the interface during capillary rise of a liquid) depending on time and the characteristic dimensions of a porous medium [10, 12]. However, the effect of various factors on the growth dynamics of interfaces at the final stage of formation before pinning has been poorly studied experimentally. Such studies 


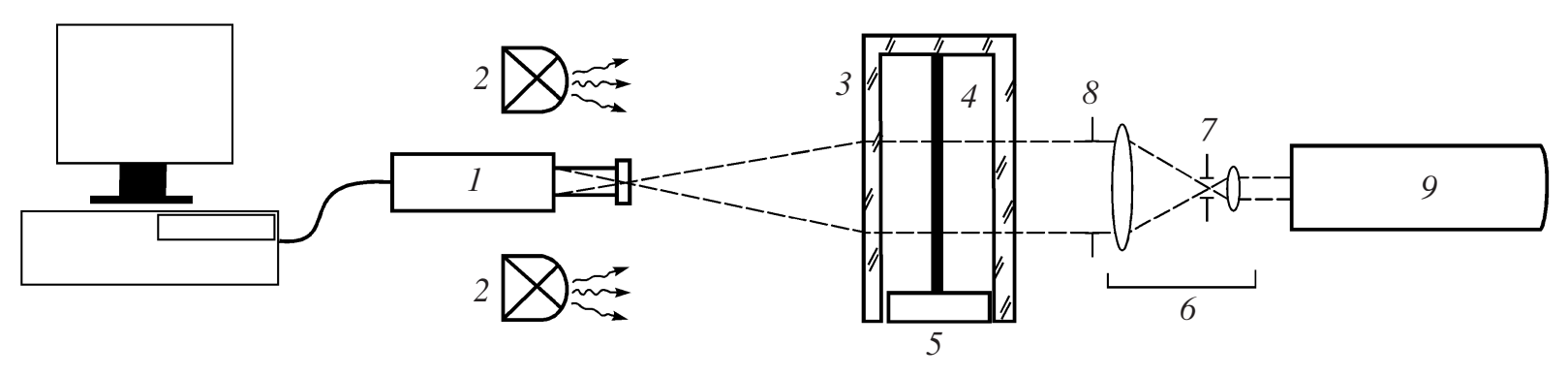

Fig. 1. Scheme of the experimental setup for investigating the critical behavior of phase interfaces in porous layers with the use of a projection method under noncoherent illumination and the speckle-correlation analysis; (1) CCD camera, (2) luminescent lamps for noncoherent illumination, (3) container with plane-parallel glass walls, (4) sample under test, (5) vessel with a liquid, (6) telescopic system (collimator and expander for a laser beam), (7) pinhole aperture (spatial filter), (8) aperture confining the cross section of the incident beam, and (9) GN-5P helium-neon laser.

are of interest not only for further developing the physics of the critical state, but also for solving a number of practical problems related to nonstationary mass transfer in disordered media (for example, monitoring the transport of biologically active substances in biological tissues).

In the present paper, we report the results of experimental investigation into the critical behavior of the interface between liquid and gaseous phases in disordered porous media with the use of noncoherent and coherent radiation incident on the interface. In the latter case, the scattering of laser radiation by dynamic irregularities (local phase interfaces in pores in unstable regions of the global interface) is responsible for the dynamic speckle-modulation of images of an object. A correlation analysis of the intensity fluctuations of the speckles that modulate the images makes it possible to describe the evolution of an ensemble of local instabilities of the interface as $f \longrightarrow 0$. A similar approach was applied earlier to analyzing the dynamics of local phase interfaces in pores during evaporation of a liquid from porous layers [13].

\section{EXPERIMENTAL METHOD AND A METHOD FOR PROCESSING THE RESULTS}

We investigated the growth dynamics of an interface between liquid and gaseous phases in porous layers during capillary rise of a liquid by a projection method under noncoherent illumination of samples and speckle-correlation analysis of the total field. In the first case, we detected sequences of images of the surface of a porous layer under diffuse illumination by broadband light sources (luminescent lamps). For the speckle-correlation analysis, speckle-modulated images of the surface of a layer were formed by laser radiation scattered forward at small angles when a collimated laser beam with a uniform intensity distribution was incident on the layer. Figure 1 shows the experimental setup, which implements both methods. Sequences of images of the layer surface near the phase interface were detected by a VS-CTT-075-2000 monochrome CCD camera (the number of pixels in the CCD sensor is $782 \times 582$, eight bits per pixel, and the linear size of a pixel is $8.3 \times$ $8.3 \mu \mathrm{m}$ pixels). The optical system (National CC TV objective lens) formed a reduced image of the sample surface on the CCD sensor. When studying the growth of local instabilities of the phase interface under noncoherent illumination, we used a linear magnification of $0.2^{\times}$. When studying the dynamics of the global phase interface under noncoherent illumination and in the case of speckle-correlation measurements under coherent illumination, we used a magnification of $0.048^{\times}$. The larger magnification in the first case was chosen because the characteristic size of instabilities at the initial stage of growth was no greater than $0.10-0.15 \mathrm{~mm}$ in all samples. The diameter of the lens aperture for recording speckle-modulated images was chosen so that the average size of the speckles approximately corresponded to the pixel size of the CCD camera.

In diffuse reflected noncoherent and transmitted coherent light, the image sequences of the sample surfaces were recorded as follows: the CCD camera was activated by a control program, supplied by SObI Videoskan NPK together with the camera, in the video data display mode with a rate of 12.5 frames/s in a window of $300 \times 300$ pixels. Since the control program did not allow storage on a hard disk with a fixed frame-toframe interval, we used SnagIt (version 8.0, TechSmith, www.techsmith.com) for real-time capture of video data in a chosen window and recording on a hard disk in the off-line mode. To prevent distortion of video data (which is especially important for correlation analysis of speckle-modulated images), we used a video capture mode without compression; in the time-lapse capture mode, we chose the same frame-repetition rates for capture and reproduction of video data, which were equal to the frame-repetition rate of the CCD camera. For further analysis, the data stored by the utility in *.avi format were converted into a sequence of bitmapped images (in *.bmp format) with the use of a specially designed program in the MatLab 6.0 environment. A similar procedure was used earlier, in particular, in the speckle-correlation analysis of a thermal modification of collagenous tissues [14]. Before carrying out experiments, we tested the setup by spectral 
analysis of sequences of images of a uniformly moving graphics test-object in the form of a system of parallel strips with a sinusoidal grayscale distribution. The analysis showed that, for detection modes satisfying the conditions of the sampling theorem [15], the detected brightness oscillations do not show any appreciable deviations from the expected harmonic law, due to the procedure used.

Since the maximal height of a liquid in a layer in our experiments was much greater than the size of the field of view of the objective lens-CCD camera system in the vertical direction, the rise of the interface was recorded fragmentarily, by timing each sequence of images to the starting point of the process and referring the position of the system to the vertical direction (with respect to the level of the liquid in vessel 5; see Fig. 1). Between the write cycles of fragments, we changed the positions of the CCD camera and the objective lens in the vertical direction and transferred the video data accumulated by SnagIt to a hard disk. The rack mechanism for moving the CCD camera with the objective lens was equipped with a scale that gave a readout of their position with an accuracy of at least $0.025 \mathrm{~mm}$. The starting and end instants of recording of the fragments were fixed by computer timer with respect to the starting time of rise of the liquid in a layer. When adjusting the setup, we paid special attention to the uniformity of illumination of the surface of samples under both noncoherent and coherent illumination. In the case of noncoherent illumination, the uniformity was provided by a symmetric arrangement of luminescent lamps with respect to the sample container. In the case of coherent illumination, to form a collimated sounding beam with a uniform intensity distribution in the paraxial domain with a diameter of at least $120 \mathrm{~mm}$, we used a telescopic system with a $40^{\times} \times 0.65$ microobjective lens with an aperture (a spatial filter of diameter of $10 \mu \mathrm{m}$ ) and a collimating lens (with a diameter, determined by a beam-confining aperture, of $150 \mathrm{~mm}$ and a focal range of $800 \mathrm{~mm}$ ).

To choose the number of fragments (video clips), their duration, and the approximate starting time of recording for each fragment at the stage of planning an experiment, we used the results of a preliminary analysis of the interface dynamics obtained from the data of preliminary experiments carried out for each sample. In the preliminary experiments, we detected relatively short (with a constant duration of $20 \mathrm{~s}$ ) sequences of images with constant spacing of $3 \mathrm{~min}$. For a detailed analysis of the behavior of the interface during its growth, at the next stage of collecting experimental data, we gradually increased the length of fragments as the interface rose from $10 \mathrm{~s}$ at the initial stage of growth to 4-7 min (depending on a sample) at the final stage of interface pinning. Preliminary analysis of the experimental data showed that the Lucas-Washburn model [16] is an approximation with acceptable accuracy (see below) at the stage preceding the interface pinning. This allowed us to record the fragments at this stage without partial overlapping of the record regions for successive fragments, followed by interpolation of the average velocity of the interface for time intervals between the end and beginning of fragments. Such a method of recording initial data was chosen primarily due to the restricted system and software resources, which did not allow the capture of large volumes (more than 2 GB) of video data.

When processing image sequences under noncoherent illumination, the sequences of frames obtained were smoothed with the use of a median filter and then converted to a binary form. The cutoff level was chosen as $\left(\left\langle I_{\text {dry }}\right\rangle+\left\langle I_{\text {wet }}\right\rangle\right) / 2$, where $\left\langle I_{\text {dry }}\right\rangle$ and $\left\langle I_{\text {wet }}\right\rangle$ are the mean values of the brightness of image fragments in dry and wet regions of the porous layer near the phase interface. The size of the fragments analyzed for determining $\left\langle I_{\text {dry }}\right\rangle$ and $\left\langle I_{\text {wet }}\right\rangle$ was chosen as $20 \times 20$ pixels. Then, the binarized images $\left\{\delta^{k}(i, j)\right\}\left(\delta^{k}(i, j)=1\right.$ in a wet region of the layer and $\delta^{k}(i, j)=0$ in a dry region, where $k$ is the frame number in a sequence and $i$ and $j$ are the numbers of columns and rows in the frame) of the layer surface, were used (a) for preliminary analysis of the data obtained during preliminary experiments with the test samples to determine the number and the length of the image sequences necessary for analyzing the behavior of the phase interface at different growth stages; and (b) for analysis of the average velocity of the phase interface as a function of the critical parameter by means of the experimental data obtained from results of preliminary experiments.

Note that, in the general case of growth of an interface between liquid and gaseous phases in thick porous layers, the distribution of the liquid near the interface is three-dimensional; however, for thin paper samples used in the experiment, the characteristic dimensions of irregularities of the interface in a direction perpendicular to its propagation are, as a rule, greater than the layer thickness. The comparison of the shape of the interface at the stage of pinning for binarized images of two surfaces of different samples, made during preliminary experiments by rotating container 3 with samples (see Fig. 1) through $180^{\circ}$, showed that these images are nearly identical. The difference in the vertical positions of the interface for the same value of the longitudinal coordinate (perpendicular to the preferred direction of growth of the interface) measured from the boundary of a layer was no greater than 1-3 pixels, which can be attributed to small defocusing of the recorded image due to rotation, to the difference in the sensitivity of the pixels of the CCD camera, and to other factors. Thus, in further analysis we assumed that the distribution of the liquid phase in the domain of growth of the interface is quasi-two-dimensional.

For the sequences of binarized images obtained in the preliminary experiments, we determined the average height of the upper boundary of the interface, $\bar{h}_{\text {max }} \approx K \sum_{i} j_{\max }(i)_{\delta=1} / \hat{L}$, as a function of time $t=$ $t_{\mathrm{st}}+k \Delta T$. Here, $j_{\max }(i)_{\delta=1}$ is the maximal row number for 
the $i$ th column in the binarized image with $\delta^{k}(i, j)=1$, $\hat{L}$ is the number of columns in the image, $K$ is the scaling factor depending on the magnification of the optical system and on the pixel size of the CCD sensor (in our case, $K=173 \mu \mathrm{m}), t_{\mathrm{st}}$ is the time interval between the start of capillary rise of a liquid and the start of recording a video clip, and $\Delta T$ is the recording frame-to-frame interval, $83 \mu \mathrm{s}$. Using these values of $\bar{h}_{\max }(t)$, we then determined approximate values of fragment lengths at the stage of final collection of data as a function of $t_{\mathrm{st}}$ :

$$
T_{\mathrm{fr}}\left(t_{\mathrm{st}}\right)=\frac{20 \cdot 0.9 H_{\|}}{\bar{h}_{\max }\left(t_{\mathrm{st}}+20\right)-\bar{h}_{\max }\left(t_{\mathrm{st}}\right)} .
$$

Here, $H_{\|}$is the vertical size of the field of view, the domain of analysis is assumed to be 0.9 of the vertical size, and the length of the fragments recorded for preliminary analysis is $20 \mathrm{~s}$. Note that, as applied to the fractal interfaces between liquid and gaseous phases that grow in porous layers and admit the existence of nonconvex regions (for example, when $\delta^{k}\left(i, j_{\max }(i)\right)=1$, $\delta^{k}\left(i, j^{\prime}(i)\right)=1$, and $\delta^{k}(i, j)_{j^{\prime}<j<j_{\max }}=0$ in a binarized image for some group of columns $\left.i^{\prime} \leq i \leq i^{\prime \prime}\right)$, this algorithm gives slightly overstated estimates for the average height of the interface. Therefore, at the final stage of data analysis, we estimated the average velocity of the interface by an algorithm that determines the increment of a liquid-saturated surface area of a layer within a given interval rather than by the formula $\left[\bar{h}_{\max }\left(t_{\mathrm{st}}+\right.\right.$ $\left.k \Delta T)-\bar{h}_{\max }\left(t_{\mathrm{st}}\right)\right] / k \Delta T$. For two binarized images of the interface that correspond to time instants $t_{\mathrm{st}}+k_{2} \Delta T$ and $t_{\mathrm{st}}+k_{1} \Delta T$, the average velocity of interface as a function of time $t=t_{\mathrm{st}}+\Delta T\left(k_{1}+k_{2}\right) / 2$ was determined as

$$
\bar{V} \approx K \frac{\sum_{i, j} \delta^{k_{2}}(i, j)-\sum_{i, j} \delta^{k_{1}}(i, j)}{\hat{L}\left(k_{2}-k_{1}\right) \Delta T} .
$$

One can easily verify that, in the case of a quasitwo-dimensional distribution of a liquid in a layer, the calculation of the increment of the area of a liquid-saturated region of the layer makes it possible to adequately estimate the increase in the liquid volume in the interface growth region within time $\Delta T\left(k_{2}-k_{1}\right)$ even when the binarized image contains nonconvex regions. Using the values of $\bar{V}$ obtained in the experiment and determined by the interpolation of time intervals between image sequences, we reconstructed the average interface height $\bar{h}(t)$ by numerically integrating the expression

$$
\bar{h}(t) \approx \int_{0}^{t} \bar{V}\left(t^{\prime}\right) d t^{\prime} .
$$

Comparison of these values of $\bar{h}(t)$ with $\bar{h}_{\max }(t)$ showed that the maximal difference $\bar{h}_{\max }(t)-\bar{h}(t)$ at the stage of interface pinning did not exceed $1.0 \mathrm{~mm}$ for all the test samples.

When analyzing binarized images under noncoherent illumination, we also considered the average number $\bar{N}_{\text {aval }}$ of local instabilities of the front (avalanches), which is determined as the number of nonoverlapping domains in the difference image $\delta^{k_{2}}(i, j)-\delta^{k_{1}}(i, j)$ as a function of $t=t_{\mathrm{st}}+\Delta T\left(k_{1}+k_{2}\right) / 2, \Delta t=\left(k_{2}-k_{1}\right) \Delta T$, and $\bar{h}$.

In addition, using specially designed software in the MatLab 6.0 environment, we analyzed the dynamics of individual local instabilities at the final stage of rise of a liquid in a layer and averaged the results of the analysis over an ensemble of instabilities that arise and grow within the time interval from $t_{\mathrm{st}}+k_{1} \Delta T$ to $t_{\mathrm{st}}+k_{2} \Delta T$. In this case, the magnification of the optical system was $0.2^{\times}$and the scaling factor was $41.5 \mu \mathrm{m} /$ pixel.

The sequences of speckle-modulated images of the surface of a porous layer obtained under coherent illumination were processed by calculating the local values of the normalized autocorrelation function of intensity fluctuations, $g_{2}(i, j, t, \Delta k)=G_{2}(i, j, t, \Delta k) / G_{2}(i, j, t, 0)$, where

$$
\begin{gathered}
G_{2}(i, j, t, \Delta k)=\sum_{k=k_{1}}^{k_{2}}\left[I^{k+\Delta k}(i, j)-\bar{I}(i, j)\right] \\
\times\left[I^{k}(i, j)-\bar{I}(i, j)\right], \\
\bar{I}(i, j)=\sum_{k=k_{1}}^{k_{2}} \frac{I^{k}(i, j)}{k_{2}-k_{1}}, \quad \Delta k \ll k_{2}-k_{1}, \\
t=t_{\mathrm{st}}+\frac{\Delta T}{2}\left(k_{1}+k_{2}\right) .
\end{gathered}
$$

Using these values of $g_{2}(i, j, t, \Delta k)$, we calculated the local values of the first cumulant of the correlation function of intensity fluctuations $[17,18]$ :

$$
\Gamma_{1}(i, j, t)=\ln \left[g_{2}(i, j, t, \Delta k=1)\right] / \Delta T .
$$

As porous layers with disordered fibrillar structure, we used samples of filter paper labeled as FM (moderate filtering, "white band"), FS (slow filtering, "blue band"), and FF (fast filtering, "red band") according to GOST 12026-76. As a saturating liquid, we used distilled water. Paper strips $150 \mathrm{~mm}$ long with a width ranging from 35 to $60 \mathrm{~mm}$ were placed vertically in a fixing frame so that the lower margin of a sample is in contact with the saturating liquid, whose level in vessel 5 (see Fig. 1) was kept constant. To reduce the effect of evaporation of the liquid from the surface of a porous layer on the growth of phase interface, we placed the fixing frame and the vessel in a closed glass container with plane-parallel glass walls. For each type of filter 


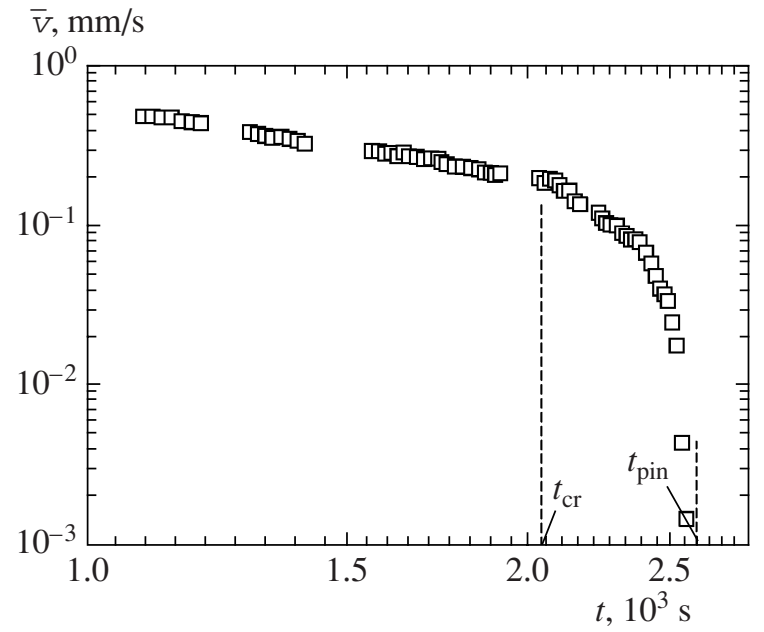

Fig. 2. Average velocity $\bar{V}$ of interface as a function of time for an FM sample.

paper, we carried out ten series of measurements both under noncoherent and coherent illumination with the view of subsequent statistical processing.

\section{RESULTS AND DISCUSSION}

\subsection{Macroscopic Dynamics of the Interface for Small and Large Times of Capillary Rise}

Figure 2 represents the time dependence of the experimental average velocity $\bar{V}$ of the boundary of a liquid-saturated layer for one of the test samples (filter paper FM). This function shows that there exist two characteristic modes of stationary flow of a liquid in a porous layer under capillary forces. When $t<t_{\mathrm{cr}}$, the data obtained admit a high-accuracy exponential approximation $\bar{V}(t) \propto \exp \left(-t / t_{\text {rel }}\right)$, where $t_{\text {rel }}$ is a characteristic time determined by the structural characteristics of the porous layer. Many authors (see, for example, [19]) pointed out that, at its early stage, the capillary rise of a liquid phase in a porous layer can be adequately described by the Lucas-Washburn model [16] with appropriately chosen parameters. The LucasWashburn equation, which describes the dynamics of rise of a liquid in an isolated capillary, is expressed as

$$
A t=-B h(t)-\ln [1-B h(t)],
$$

where $h(t)$ is the current height of the liquid in a capillary; $A=\rho^{2} g^{2} r^{3} / 16 \sigma \eta \cos \vartheta ; B=\rho g r / 2 \sigma \cos \vartheta ;$ and $\rho, \eta$, and $\sigma$ are, respectively, the density, viscosity, and surface tension of the liquid; $r$ is the capillary radius; and $\vartheta$ is the contact angle determined by the interaction between the liquid and the capillary walls. For a capillary rise of a liquid with known $\rho, \eta$, and $\sigma$ in a porous layer, the approximation of the experimental dependence $\bar{h}(t)=\int_{0}^{t} \bar{V}(\tau) d \tau$ with the use of Eq. (3) allows

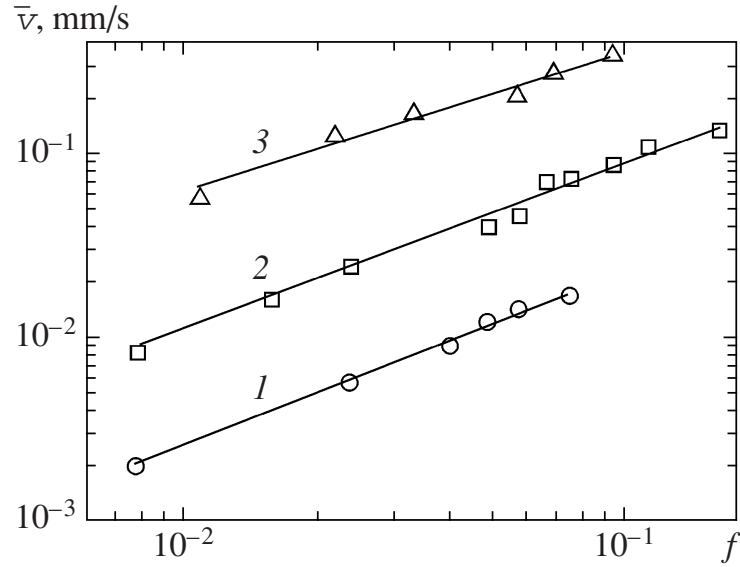

Fig. 3. $\bar{V}$ as a function of the critical parameter $f$ at the stage of interface pinning for different samples; (1) FS, (2) FF, and (3) FM.

one to determine the effective value of the capillary radius $r_{\text {eff }}$, which is determined by the characteristic size of pores in the layer and the porosity of the layer, and the value of $\cos \vartheta$.

As $t \longrightarrow \infty$, the Lucas-Washburn equation describes the asymptotic behavior of the interface, which tends to an equilibrium state, $\bar{h}(t)_{t \rightarrow \infty} \longrightarrow \bar{h}_{\mathrm{eq}}=1 / B$, which is independent of the viscosity of the liquid. It can be shown that, within the Lucas-Washburn model, the time dependence of the absolute value of the average velocity $|\bar{V}|$ on the saturation time of a layer at large time scales is described by the expression $|\bar{V}(t)| \approx$ $(A / B) \exp (-A t)$, which corresponds to the experimentally observed behavior of the phase interface in all test samples for $t<t_{\mathrm{cr}}$ (see Fig. 2). A sharp decrease in $\bar{V}$ for $t>t_{\mathrm{cr}}$ corresponds to the critical interface pinning mode as $f \longrightarrow 0$, which is characterized by a power law dependence of the average velocity of interface growth on the critical parameter: $\bar{V} \propto f^{\theta}$. Note that the values of $\bar{h}_{\text {pin }}$ determined in the experiment for the pinned interface in porous layers are substantially less than the equilibrium values of the rise height of the liquid, which are determined by the Lucas-Washburn model for $t \longrightarrow \infty: \bar{h}_{\text {pin }} \ll \bar{h}_{\text {eq }}[20]$.

Figure 3 shows the values of $\bar{V}$, measured with the use of a projection method under noncoherent illumination, as a function of the critical parameter at the stage of interface pinning in the samples; the values of $f$ were determined from the current value of $\bar{h}(t)$ and the value of $\bar{h}_{\text {pin }}$ measured as $t \longrightarrow \infty$ for the pinned interface: $f \approx \bar{h}_{\text {pin }} / \bar{h}(t)-1$ [20]. All the experimental functions $\bar{V}(f)$ admit a power law approximation of reasonable accuracy as $f \longrightarrow 0$, which is indicative of the critical 
Critical indices $\theta$ transition times to the critical mode $\left(t_{\mathrm{cr}}\right)$ and to the interface pinning mode $\left(t_{\text {pin }}\right)$; and the height $\bar{h}_{\text {pin }}$ of a pinned interface for test samples (results of statistical processing over ten series of experimental data for each type of paper; the significance level is 0.9 )

\begin{tabular}{c|c|c|c|c}
\hline Sample & $\theta$ & $t_{\mathrm{cr}}, \mathrm{s}$ & $t_{\mathrm{pin}}, \mathrm{s}$ & $\bar{h}_{\mathrm{pin}}, \mathrm{mm}$ \\
\hline FF & $0.89 \pm 0.06$ & $2150 \pm 110$ & $3300 \pm 170$ & $127.4 \pm 3.6$ \\
FM & $0.76 \pm 0.07$ & $2050 \pm 106$ & $2600 \pm 135$ & $93.0 \pm 2.9$ \\
FS & $0.93 \pm 0.07$ & $1800 \pm 98$ & $2900 \pm 150$ & $64.5 \pm 1.7$ \\
\hline
\end{tabular}

behavior of the phase interface. The table presents the values of $\theta, t_{\mathrm{cr}}, t_{\mathrm{pin}}$, and $\bar{h}_{\mathrm{pin}}$ for the test samples.

The values of $\theta \leq 1$ correspond to a finite duration of the interface pinning process under capillary rise of a liquid in thin disordered porous layers. Note that the experimental data presented in [20] on the growth of the interface under capillary rise of a liquid in a cylindrical porous column of diameter $8 \mathrm{~mm}$ consisting of densely packed glass spheres with diameters of 180 , 253,359 , and $510 \mu \mathrm{m}$ are evidence for a different scenario of the critical behavior of the phase interface. In particular, the value of $\theta$ for a porous medium, evaluated according to the experimental data, is substantially greater than unity, which corresponds to an infinitely large pinning time of the phase interface. At larger time scales, one can observe a power law decay of $\bar{V}$ as a function of $t$ with an exponent of about 0.75 (according to the experimental data, the average velocity of the interface exhibits power law time dependence in a time interval of more than three orders of magnitude). It is noteworthy that the porous system studied in [20] is characterized by a higher degree of structural ordering and greater dimension compared with our samples: the phase interface cannot be represented by a fractal line like in our case (a quasi-two-dimensional flow of a liquid in a thin porous layer); it is, rather, a surface (threedimensional flow of a liquid).

The transfer of the liquid phase in paper layers strongly depends on the interaction between the saturating liquid and cellulose fibers (for example, swelling due to saturation of a paper layer with water). Therefore, the effect of interaction between the saturating liquid and the hydrophilic fibers that form the porous layer on the growth of the phase interface in the layer requires a more comprehensive analysis. The authors of [21] point to two basic factors that determine the process of saturation of paper layers with water: the absorption of water by the fiber surface and the swelling of fibers. Due to the formation of an additional channel for mass transfer in a porous medium and due to a change in the dimensional characteristics of the system of pores in time and over the bulk of the medium, these factors may affect the growth of the global phase interface in a porous layer. During the initial stage of rise of the liquid in the layer, which is described by the Lucas-Washburn equation, these fac- tors should lead to a nontrivial time dependence of the model parameters $A$ and $B$ (expression (3)) and, consequently, to a slightly different form of the function $\bar{h}(t)$ compared with the classical Lucas-Washburn equation with $A(t)=$ const and $B(t)=$ const. In [21], the authors present experimental data on the swelling kinetics of cellulose fibers during hydration, which make it possible to evaluate the characteristic time of this process as $40-50 \mathrm{~s}$; the maximal increase in the fiber diameter is no greater than $15 \%$. Assuming that the average size of pores during the hydration of cellulose fibers changes by a value on the same order of magnitude and taking into account that the effective capillary radius $r$ in the Lucas-Washburn model depends on the average size of pores and the porosity of a layer, we can predict a slight variation (by about 10-20\%) $\mathrm{m}$ the equilibrium height $\bar{h}_{\text {eq }}$ of the liquid compared with the stationary LucasWashburn model with $A(t)=$ const and $B(t)=$ const.

The experimental functions $\bar{h}(t)$ and $\bar{m}(t)$ given in [21], where $\bar{m}$ is the current value of the mass of the liquid in the layer, at early stages of rise of the liquid in paper layers admit a high-accuracy approximation of the form $\bar{h}(t) \propto \sqrt{t}, \bar{m}(t) \propto \sqrt{t}$, which is predicted by the Lucas-Washburn model for small rise times; however, the slope of the curve $\bar{m}(t)$ is smaller than that of $\bar{h}(t)$ in the coordinates $(\bar{h}, \bar{m} ; \sqrt{t})$. This difference is attributed to the effect of the characteristic hydration time of cellulose fibers whereby the degree of saturation of cellulose fibers in the regions immediately below the phase interface turns out to be less than the limit value. Note also that the propagation of a liquid in porous media depends on macroscopic irregularities of the medium, which lead to the distortion of the phase interface near the irregularity area [22].

It is obvious that the structural characteristics of a porous medium, just like the parameters of interaction between the liquid and the substance forming the porous structure, have a significant effect on the formation of the interface as $\bar{h} \longrightarrow \bar{h}_{\text {pin }}$, which is indicated by a significant difference between the values of $\bar{h}_{\text {pin }}$ for the samples of FF, FM, and FS. The data in the table, combined with the structural characteristics of the samples, imply that a decrease in the permeability of a 


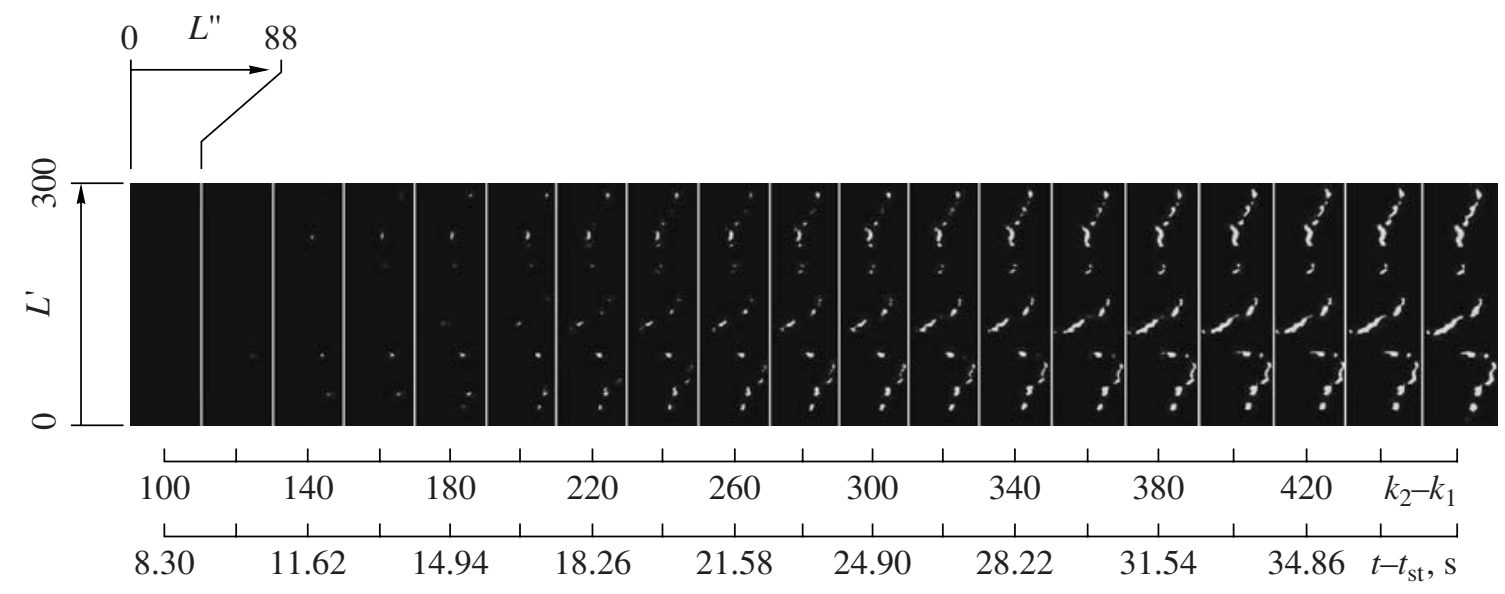

Fig. 4. Growth of local instabilities at the stage of interface pinning. FM sample, $f \approx 0.1$. Linear magnification of the optical system is $0.2^{\times} . L^{\prime}$ and $L^{\prime \prime}$ are measured in the number of pixels.

porous layer leads to a decrease in $\bar{h}_{\text {pin }}$. However, we should not think that the interface pinning as $\bar{h} \longrightarrow$ $\bar{h}_{\text {pin }}$ is attributed precisely to the interaction between the liquid and the material of the porous medium (interaction between water and cellulose fibers). First of all, we should note that $\bar{h}_{\text {pin }} \ll \bar{h}_{\text {eq }}$, and variations in the equilibrium height, anticipated as a result of hydration, do not exceed $15-20 \%$.

In $[8,10,11]$, using computer simulation methods, the authors investigated the formation of a phase interface in disordered porous media by two- and threedimensional stochastic grid models that do not take into account how filling of the grid cells affect their parameters. Nevertheless, similar model systems exhibit critical behavior of their characteristics (including the behavior of the average velocity of propagation of the global interface) as the control parameter tends to zero. Investigations of the propagation of the interface between liquid and gaseous phases in three-dimensional media formed by hydrophilic ceramic materials (see, for example, [10]), in which phenomena like the swelling of cellulose fibers in paper are completely excluded, are also evidence for the critical behavior and fractal properties of the developing interface at small propagation velocities. These phenomena cannot be described within continual concepts about fluid transfer in porous media (see, for example, [22]).

Thus, the critical behavior of interfaces and the pinning effect as $f \longrightarrow 0$ in disordered porous systems are a fundamental property of these systems, which is associated precisely with the structural disorder of a system rather than with the variations in the structure and the properties of the system due to interaction with the liquid phase. Note also that, in paper layers at the stage of interface pinning, rather slow processes of hydration and swelling of fibers immediately below the interface may substantially affect the kinetics of the appearance of local instabilities (avalanches), thus affecting the value of the critical index $\theta$. However, the saturation of these regions with a liquid phase up to the maximal concentration occurs in a finite time [21]; for paper layers, this rules out the possibility that a phase interface will exhibit behavior similar to that described in [20].

We can propose the following qualitative interpretation for the significant difference in the critical behavior of two types of porous systems: in the case of disordered systems considered in the present study, when the velocity of the phase interface reaches a certain value $\bar{V}_{\text {ch }}$ characteristic of the medium, continuous motion of the phase interface described by the Lucas-Washburn model is suppressed and it changes to discrete decaying motion controlled by the appearance, growth, and decay of local instabilities (avalanches). In the case of a porous system with a higher degree of structural ordering [20], the continuous motion of the phase interface presumably is not completely suppressed; however, for average velocities of the interface lower than $\bar{V}_{\text {ch }} \approx 100 \mu \mathrm{m} / \mathrm{s}$, an increase in the intensity of spacetime fluctuations of a local velocity $\mathrm{v}(x, t)$ leads to a power law time dependence at large time scales (which was obtained in [20]), rather than to the critical behavior.

\subsection{Microscopic Dynamics of the Interface} at the Pinning Stage (Results of Analysis of Binarized Images under Noncoherent Illumination)

As $f \longrightarrow 0$ and, accordingly, $\bar{h}(t) \longrightarrow \bar{h}_{\text {pin }}$, the motion of the phase interface represents the sequence of appearance, growth, and decay of local instabilities of the interface. Figure 4, which presents a sequence of binarized difference images of the surface of a sample in the localization region of the phase interface, illustrates the evolution of an ensemble of localized instabilities of the interface for small values of the critical parameter. Each of the 19 fragments of this image, sep- 


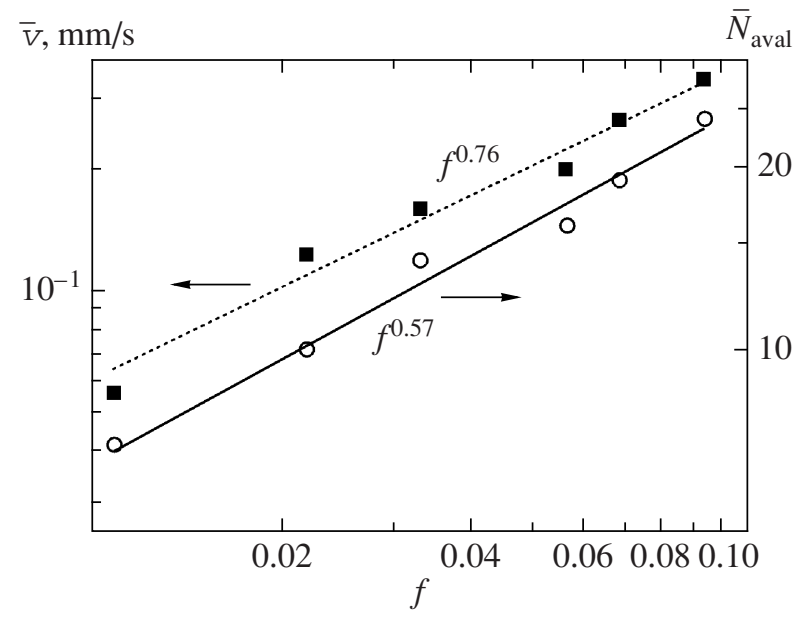

Fig. 5. $\bar{N}_{\text {aval }}$ and $\bar{V}$ as a function of the critical parameter $f$ at the stage of interface pinning for an FM sample.

arated by lines, represents a region of a difference image of a localization domain of the phase interface obtained for different values of $k_{2}-k_{1}$ (an increment of $k_{2}-k_{1}$ during the image synthesis is 20 ). From considerations of compact representation of a set of difference images, we chose the vertical coordinate $L^{\prime}$ perpendicular to the propagation direction of the phase interface (to a horizontal direction in the recorded images). The size of a region in the direction of $L^{\prime}$ corresponds to the width of the field of view ( 300 pixels, or $12.45 \mathrm{~mm}$ for a linear magnification of the system of $\left.0.2^{\times}\right)$; in the direction in which the interface propagates (the vertical direction in the images of surface of a paper layer), the size of the regions was chosen according to the criterion $L_{\max }^{\prime \prime} \approx 1.1\left(h_{\max }-h_{\min }\right)_{t=t_{\mathrm{st}}}$, where $h_{\max }$ and $h_{\min }$ are the maximal and minimal values of the height of the interface within the analyzed region at the starting point at which the sequence of images was recorded. For the example shown in Fig. 4, the value of $L_{\max }^{\prime \prime}$ is 90 pixels, or $\approx 3.74 \mathrm{~mm}$.

The analysis of difference images in Fig. 4 shows that the interface remains stable during the first $9 \mathrm{~s}$ after the start of recording of a sequence of raw images; at the tenth second, a local instability with coordinates $L^{\prime \prime} \approx 86$ pixels and $L^{\prime \prime} \approx 63$ pixels arises; at the 12 th second, one can observe three growing irregularities, and, at the 15th second, seven irregularities. Further, the existing irregularities grow and merge together, and new irregularities arise in different regions of the interface; as a result, for large values of $k_{2}-k_{1}$, a considerable part of the entire phase interface is covered by zones of active growth. For example, at $k_{2}-k_{1}=480$ (for a time interval of $38.18 \mathrm{~s}$ between the images used to construct the last fragment in Fig. 4), the total area of active growth zones amounts to $55 \%$ of the interface within the region considered. Other regions of the interface remain stable for a given time interval.

In the case shown in Fig. 4, the time $t_{\mathrm{st}}$ is $2120 \mathrm{~s}$ and the critical parameter is $f \approx 0.1$. This corresponds to the transition region (see Fig. 2) from the Lucas-Washburn mode of flow of a liquid to the growth of an interface via the appearance and growth of local instabilities, which is illustrated in Fig. 4. A further decrease in the critical parameter leads to a sharp decrease in the probability that local instabilities will appear in a finite observation time, which ultimately leads to pinning of the interface.

The average velocity of the interface for small values of the critical parameter can be represented as

$$
\bar{V}(t) \approx \frac{\bar{N}_{\text {aval }}(t)}{L} \overline{\left\langle\frac{\partial S_{i, \text { aval }}(t)}{\partial t}\right\rangle},
$$

where $\bar{N}_{\text {aval }}(t)$ is the average number of intensely growing local instabilities in a region of interface having a length of $L=K \bar{L} ; S_{i \text {, aval }}(t)$ is the area covered by the $i$ th local instability at moment $t$; and the averaging $\overline{\left\langle\partial S_{i, \text { aval }}(t) / \partial t\right\rangle}$ is performed both over the ensemble of instabilities and over the time interval used to determine $\bar{V}(t)$. According to the general principles of the behavior of critical systems as $f \longrightarrow 0$ [7], we can naturally assume that, at the pinning stage, $\bar{N}_{\text {aval }}$ and $\overline{\left\langle\partial S_{i, \text { aval }} / \partial t\right\rangle}$, as a function of the critical parameter, exhibit scaling behavior,

$$
\bar{N}_{\text {aval }} \propto f^{\gamma}, \overline{\left\langle\frac{\partial S_{i, \text { aval }}}{\partial t}\right\rangle} \propto f^{\delta},
$$

and that the following relation holds between the critical indices [7] $\theta, \gamma$, and $\delta$, which characterize the behavior of the phase interface: $\theta=\gamma+\delta$.

Figure 5 shows the functions $\bar{V}(f)$ and $\bar{N}_{\text {aval }}(f)$ for an FM sample obtained by analyzing binarized difference images of the surface in the localization region of the phase interface. For the critical index $\gamma$, the function $\bar{N}_{\text {aval }}(f)$ gives an estimate of $0.57 \pm 0.05$, which is comparable with the critical index $\theta$ of the average velocity of the interface; this suggests that the critical index $\delta$ is much smaller than $\gamma$ and, hence, the effect of the critical parameter on $\overline{\left\langle\partial S_{i \text {, aval }} / \partial t\right\rangle}$ is insignificant.

The relatively small effect of $f$ on $\overline{\left\langle\partial S_{i, \text { aval }} / \partial t\right\rangle}$ is also confirmed by analysis of the growth of individual local instabilities of the phase interface. Figure 6 shows the initial regions of the function $S_{i \text {, aval }}(t)$ for arising and growing local instabilities for various values of the critical parameter; these functions are obtained by processing sequences of binarized difference images of the surface of samples in the localization region of the interface. These functions characterize the growth of a 
single local instability within a considered region and admit a piecewise linear approximation of the form

$$
S_{i, \text { aval }}\left(t^{\prime}\right)=\left\{\begin{array}{l}
0, \quad t^{\prime} \leq t_{0 i}^{\prime} \\
\alpha_{i}\left(t^{\prime}-t_{0 i}^{\prime}\right), \quad t^{\prime}>t_{0 i}^{\prime},
\end{array}\right.
$$

where the time $t^{\prime}$ is measured from the start of recording of a sequence of images, $t_{0 i}^{\prime}$ is the time interval determined by a random delay of the point at which instability appears (a value of $\left\langle t_{0 i}^{\prime}\right\rangle$, averaged over an ensemble of local instabilities, monotonically increases as $f$ decreases), and $\alpha_{i}$ is the rate of growth of the area of the layer surface covered by a local instability during the growth of this instability (at the initial stage, $\alpha_{i} \approx$ $\partial S_{i, \text { aval }} / \partial t \approx$ const; that large time scales, $\partial S_{i, \text { aval }} / \partial t$ slowly decreases, thus leading to decay of the instability). For the functions shown in Fig. 6, the velocities $\alpha_{i}$ have close values; i.e., a significant decrease in the critical parameter does not lead to significant variations in the growth dynamics of the instabilities, which were chosen randomly for the analysis (at least at the initial stage of their growth). This result agrees with the conclusion, made by comparing the experimental values of the critical indices $\gamma$ and $\theta$, that $\overline{\left\langle\partial S_{i, \text { aval }} / \partial t\right\rangle}$ weakly depends on $f$.

\subsection{Microscopic Dynamics of the Interface (Results of Speckle-Correlation Analysis)}

The dynamic speckle modulation of images of the surface of a porous layer under coherent illumination is associated with quasi-elastic scattering of light by moving local phase interfaces in the pores in the region of active growth of the boundary of the liquid-saturated bulk of the layer. The first cumulant $\Gamma_{1}(t)$ (see formula (2)) of the correlation function of intensity fluctuations of scattered radiation is related to the halfwidth of the spectrum $S(\omega)$ of intensity fluctuations, which, in turn, is determined by the mobility of the dynamic scattering centers in the medium. For example, in the case of the Lorentz spectrum $S(\omega)=(\Gamma / \pi) /\left(\Gamma^{2}+\omega^{2}\right)$ and, hence, in the case of exponential correlation function $g_{2}(\tau)=$ $\exp (-\Gamma|\tau|)$ of intensity fluctuations, which are characteristic of single scattering of light by ensembles of monodisperse Brownian particles [23], the relation between $\Gamma_{1}$ and the halfwidth $\Gamma$ of the spectrum of intensity fluctuations is given by the trivial formula $\Gamma_{1}=\Gamma$.

For single scattering of laser radiation by ensembles of moving particles, the first cumulant $\Gamma_{1}$ is inversely proportional to the characteristic time $\tau_{\lambda}$ of displacement of scattering centers to a distance on the order of the wavelength $\lambda$ of the incident radiation and directly proportional to $\sin ^{2}(\varphi / 2): \Gamma_{1} \propto \tau_{\lambda}^{-1} \sin ^{2}(\varphi / 2)$, where $\varphi$ is the angle between the wave vectors $\mathbf{k}_{\mathrm{i}}$ and $\mathbf{k}_{\mathrm{s}}$ of the inci-

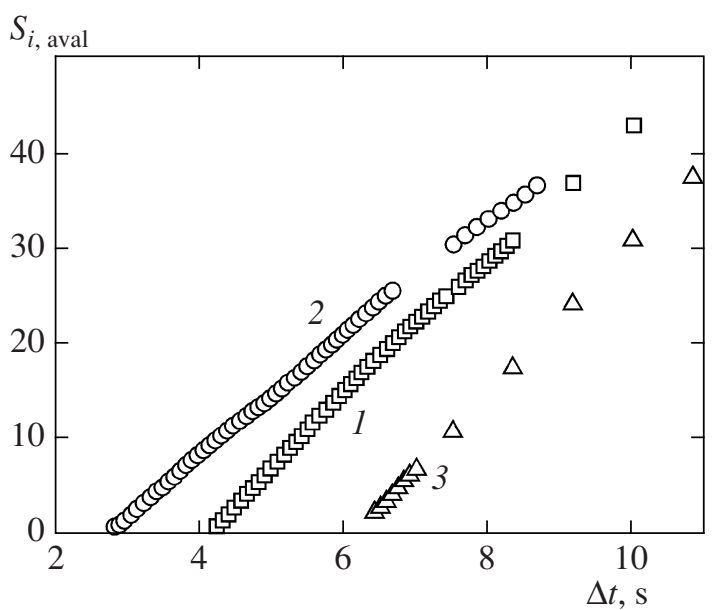

Fig. 6. Function $S_{i \text {, aval }}(\Delta t)$ for small values of the critical parameter for an FM sample; (1) $f \approx 0.07$, (2) 0.03 , and (3) 0.01 . The area is measured in the number of pixels in a region of local instability. The linear magnification of the optical system is $0.2^{\times}$.

dent beam and the detected scattered light (the scattering angle) [23]. The parameter $\tau_{\lambda}$, as a function of the mobility of scatterers, depends on the character of the scatterers' motion in the scattering medium. For example, in the case of diffuse motion, $\tau_{\lambda}=\left(D k^{2}\right)^{-1}$, where $D$ is the translation diffusion coefficient of the scatterers, $k=2 \pi n_{r} / \lambda$ is the wavenumber of incident radiation in the medium and $n_{r}$ is the refractive index of the medium. In the case of the scattering of laser radiation by an ensemble of scatterers characterized by random distribution of their velocities with a mean value $\left\langle v_{m}\right\rangle$, we have $\tau_{\lambda}=\left(\left\langle v_{m}\right\rangle k\right)^{-1}$.

In the multiple scattering regime, which should take into account the multiplicity of scattering in the expression for the first cumulant as a function of the mobility of scatterers. For approximate estimates one may assume that $\Gamma_{1} \propto\langle n\rangle / \tau_{\lambda}$, where $\langle n\rangle$ is the average multiplicity of scattering of incident radiation under fixed detection conditions. Note that, in the case of diffuse propagation of radiation, when the characteristic dimensions of the scattering medium are much greater than the transport length $l^{*}$ [24] for the medium, the parameter $\Gamma_{1}$ does not depend on the angle between the incident and detected beams; this is attributed to the nearly isotropic character of propagation of light in the medium.

Under the experimental conditions (detection of light scattered forward at small angles), the correlation time of intensity fluctuations for different regions of an image is determined by the mobility of the scattering centers (moving phase interfaces in the pores [13]) in a local volume of the porous layer under this region. The correlation time is substantially different in different regions and attains its maximum in the zones of active growth of the global phase interface in the layer. 

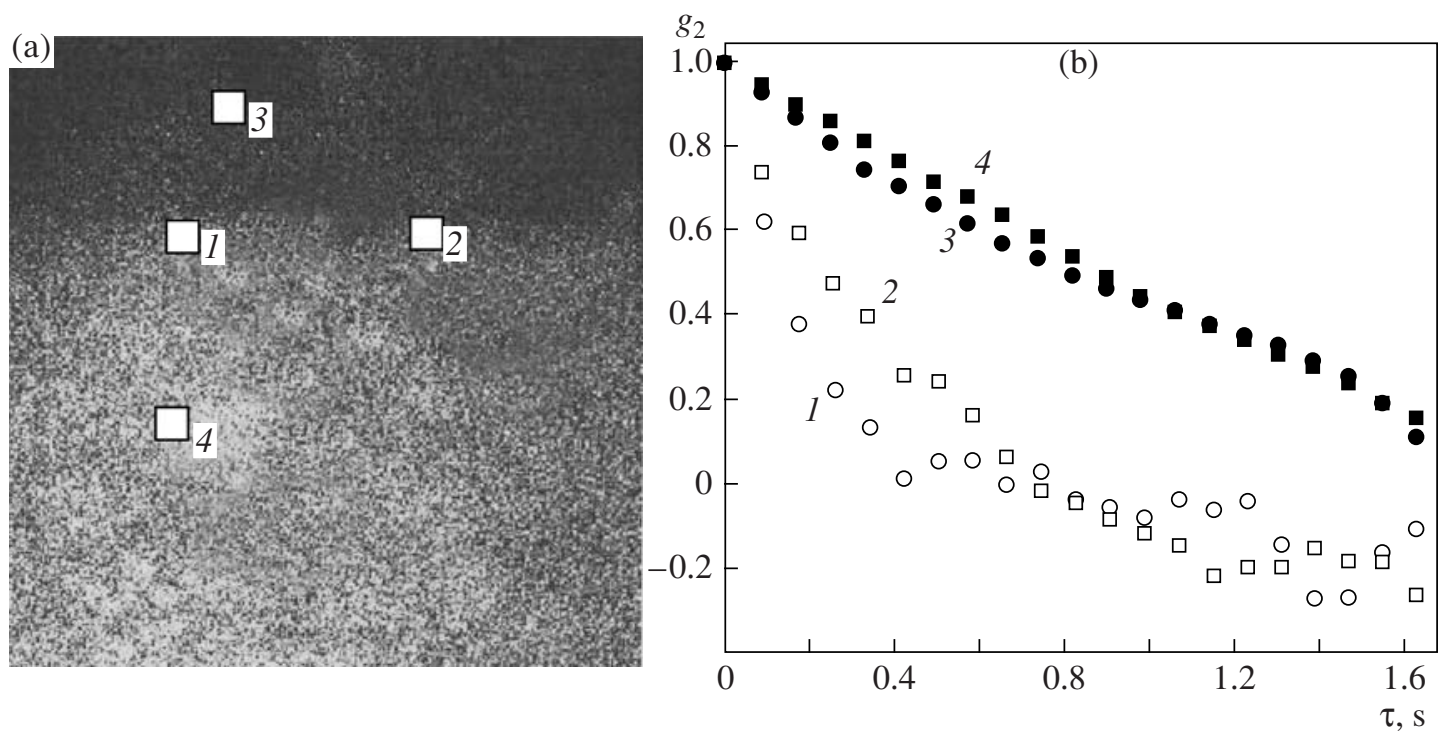

Fig. 7. (a) Speckle-modulated image of the surface of a porous layer in the growth region of the interface between the liquid and gaseous phases (linear magnification of the optical system is $0.048^{\times}$); (b) normalized time correlation functions of intensity fluctuations of speckles in different regions of the image of Fig. 7a.

Local estimates of the correlation time (or another parameter that determines the decay rate of the correlation function of intensity fluctuations) calculated at different time instants over image regions with a size on the order of an average speckle size can be used for visualizing the mobility of the liquid on a microscopic level in different active growth zones of the interface. The cumulant analysis of speckle-modulated images used in this paper is based on the local estimates of the first cumulant $\Gamma_{1}(x, y, t)$ with the use of a sliding window in the time domain over short (on the order of 40 50 frames) fragments of image sequences. The choice of the method is determined by its efficiency as applied to analysis of nonstationary dynamic speckles (especially at the stage of interface pinning, when its average velocity changes by a factor of 30-100 within times of 200-400 s; see Fig. 2).

Figure 7a illustrates substantial differences in the dynamics of speckles recorded in the growth region of the phase interface in a porous layer $(1,2)$ and outside this region $(3,4)$. Normalized correlation functions of intensity fluctuations were calculated over a time interval of $4 \mathrm{~s}$ (over 50 frames). The correlation time of intensity fluctuations estimated by the decay of the curve $g_{2}(\tau)$ down to a level of $1 / e$ (Fig. 7b) for an active growth region ranges from 0.2 to $0.4 \mathrm{~s}$, whereas for the regions 3 and 4 this time is about $1.3 \mathrm{~s}$. The decorrelation of speckles outside the active growth zone is mainly associated with the noise in the measurement system (fluctuations of the laser radiation frequency, noise of the CCD camera, and vibrations). When laser radiation is scattered in a liquid-saturated volume of a layer, processes in the layer with large characteristic times (variation in the cellulose fiber volume due to hydration) also lead to a slow decorrelation of detected speckles. The negative values of $g_{2}(\tau)$ (Fig 7b; functions 1 and 2 for $\tau>0.8 \mathrm{~s}$ ) are due to the relatively small sample size of the raw data.

Local estimates for $\Gamma_{1}(x, y, t)$ obtained by the analysis of sequences of speckle-modulated images were used for the visualization of the region of active growth. Figure 8 represents the images corresponding to the initial stage of growth, i.e., to the Lucas-Washburn mode with the viscosity of the liquid significantly affecting the rise of the liquid in the layer (Fig. 8a), and to the stage of exponential decay of the interface velocity $\bar{V}$, which precedes its pinning (Fig. 8b). The coordinate $y$ is the direction of propagation of the phase interface in the layer. When drawing these images, we calculated the first cumulant $\Gamma_{1}(i, j, y=$ const) for each pixel according to formula (2), and the normalized correlation functions $g_{2}(i, j, t=$ const, $\Delta k=1)$, by formula (1). The two-dimensional matrices of $\Gamma_{1}(i, j, t=$ const $)$ thus obtained were subjected to median filtering with a $5 \times$ 5-pixel window with a view to eliminating artifacts for individual elements of the image, which are significantly greater than the surrounding elements and are associated with noise. To give a clear representation of the active growth region of an interface and eliminate redundant details, we used four brightness grades (the appropriate scales are shown in Fig. 8).

Figure $8 \mathrm{a}$ is characterized by considerable values of the first cumulant in the zone of active growth of the interface. In Fig. 8b, the growth zone is characterized by much smaller values of $\Gamma_{1}$. Individual domains in the images outside the active growth zone are due to the above-mentioned noise in the measurement system, 

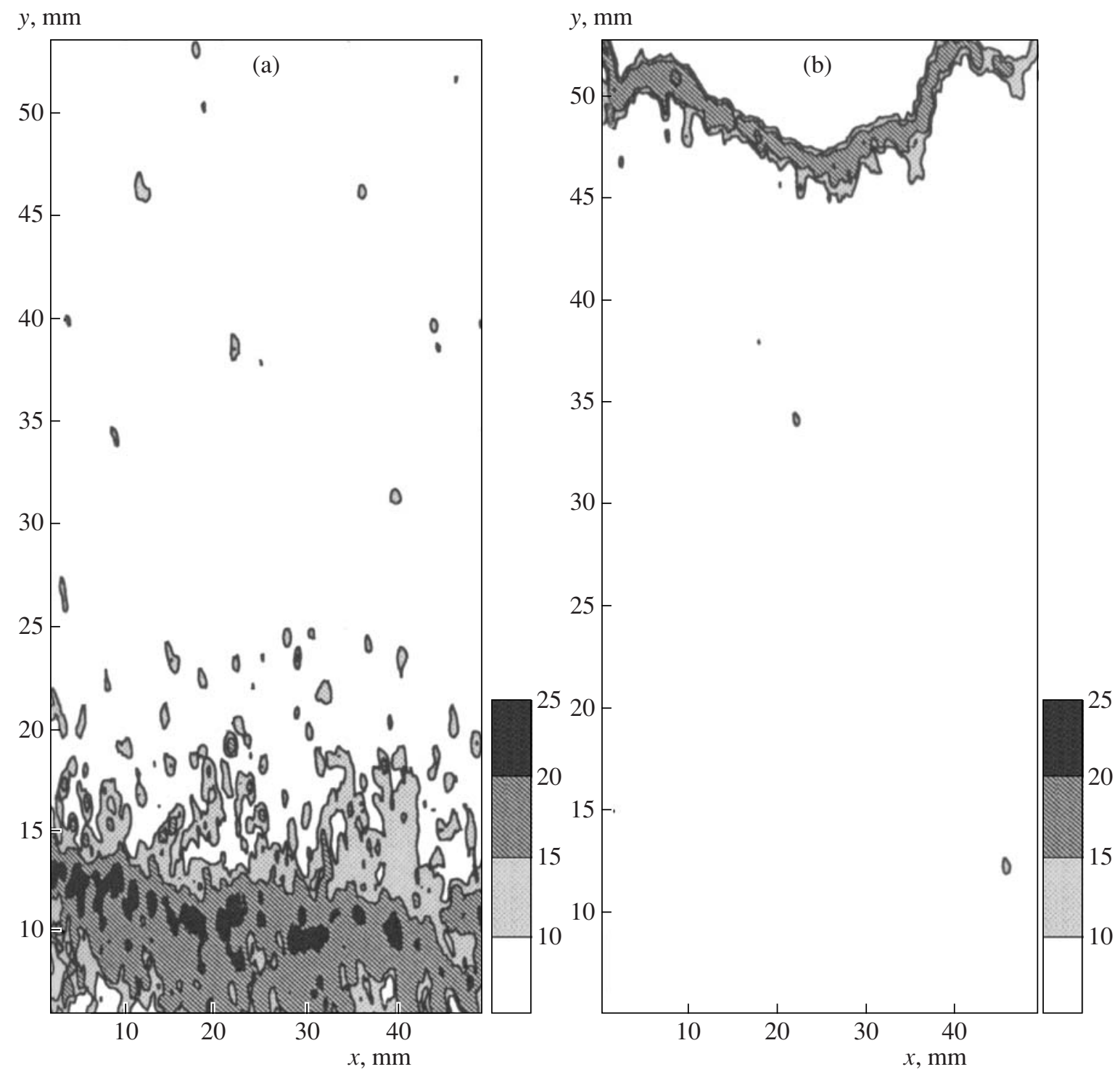

Fig. 8. Visualization of a growing interface between liquid and gaseous phases with the use of local estimates for the first cumulant $\Gamma_{1}$ as a visualization parameter; (a) the initial stage of capillary rise of a liquid; (b) a stage corresponding to exponential decay of the interface velocity as a function of time. FM sample. Linear magnification of the optical system is $0.048^{\times}$.

which leads to a slow decorrelation of speckles and determines the sensitivity threshold of the system with respect to the values of $\Gamma_{1}$. To estimate this threshold, we evaluated the average values of the first cumulant in the cases when speckle-modulated images are formed during scattering of laser radiation in the layers of dry and liquid-saturated paper. The values of $\Gamma_{1}$ were averaged over all elements of the matrix $\left\{\Gamma_{1}(i, j, t=\right.$ const $\left.)\right\}$ after median filtering. Thus, we obtained the values of $\left\langle\Gamma_{1}\right\rangle_{\text {dry }}^{\text {noise }} \approx 7.8 \mathrm{~s}^{-1}$ for dry and $\left\langle\Gamma_{1}\right\rangle_{\text {wet }}^{\text {noise }} \approx 5.2 \mathrm{~s}^{-1}$ for liquid-saturated paper. The greater threshold in dry samples, which manifests itself in faster decay of function $g_{2}(\tau)$ for small $\tau$ in region 3 compared with region 4 in Fig. 7 and in a greater number of noise fragments out- side the active growth zone in Fig. 8a, is presumably associated with stronger scattering of incident radiation in a dry layer of paper. On the one hand, this leads to a smaller value of the mean intensity of laser radiation scattered forward at small angles by a layer of dry paper compared with that scattered by liquid-saturated paper. Accordingly, for smaller values of the brightness of the recorded image, the contribution made by the noise of the CCD camera to the intensity fluctuations is more significant. In addition, a dry layer is characterized by higher multiplicity of scattering of incident radiation (in a liquid-saturated layer, multiple scattering is suppressed due to the immersion phenomenon). This should lead to an increase in the threshold value of $\Gamma_{1}$, 


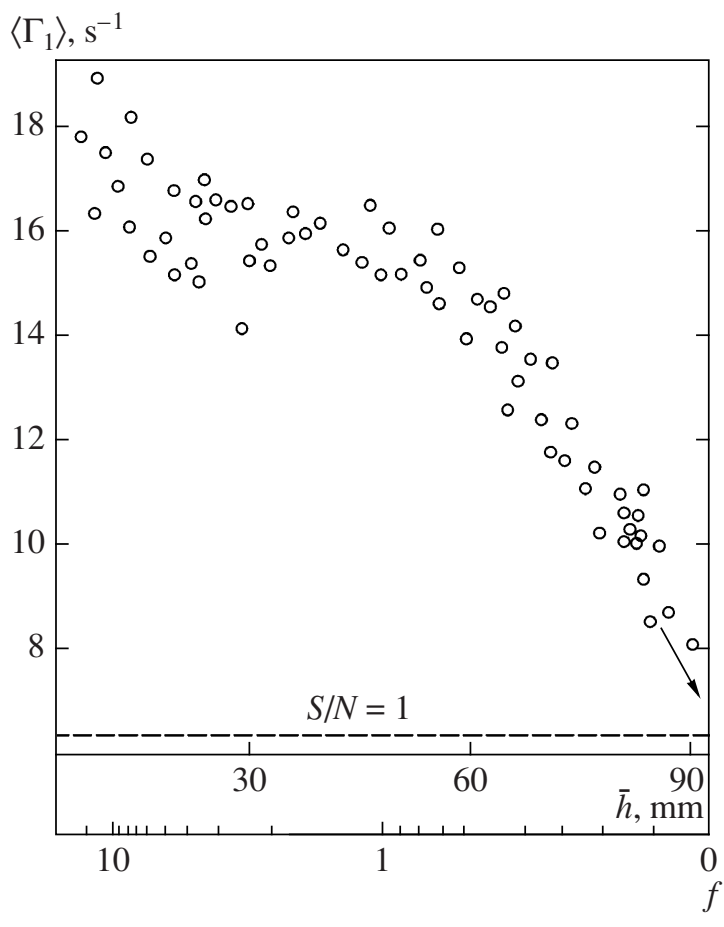

Fig. 9. $\left\langle\Gamma_{1}\right\rangle$ as a function of the average height $\bar{h}$ of the phase interface and the critical parameter $f$ for an FM sample. The dashed line shows the limit value $\left\langle\Gamma_{1}\right\rangle^{\text {noise }}$ determined by the noise of the measurement system. The arrow shows the limit state corresponding to the pinned state.

which is associated with the measurement noise and is proportional to the multiplicity of scattering.

Since the value of $\Gamma_{1}(i, j, t=$ const $)$ is determined by the characteristic time of displacement of dynamic scattering centers in a local volume of a porous layer by a distance of $\lambda$,

$$
\Gamma_{1}(i, j, t=\text { const })=C_{C} / \tau_{\lambda}(i, j, t=\text { const }),
$$

where $C_{C}$ is a dimensionless normalization factor for the cumulant method used, the averaging of $\Gamma_{1}(i, j, t=$ const) over the active growth zone of the phase interface in the layer makes it possible to characterize the mean mobility of the dynamic scatterers (phase interfaces in individual pores) for a given growth stage of the interface. When calculating the mean values $\left\langle\Gamma_{1}(t)\right\rangle$, summation was performed only over those elements of the matrix $\left\{\Gamma_{1}(i, j, t)\right\}$ whose values were greater than the threshold $\left\langle\Gamma_{1}\right\rangle^{\text {noise }}$ chosen equal to $\left(\left\langle\Gamma_{1}\right\rangle_{\text {dry }}^{\text {noise }}+\left\langle\Gamma_{1}\right\rangle_{\text {wet }}^{\text {noise }}\right) / 2$ :

$$
\left\langle\Gamma_{1}(t)\right\rangle=\frac{1}{N_{\text {th }}(t)} \sum_{i, j}^{N_{\text {th }}(t)} \Gamma_{1}(i, j, t) .
$$

The analysis of $\left\langle\Gamma_{1}\right\rangle$ as a function of $\bar{h}$ makes it possible to consider variations in the microscopic mobility of local instabilities of the interface at different stages. Figure 9 presents this function for one of the samples (filter paper FM). Note that, at the initial stage of growth, when the average velocity of the interface is rather high and the growth of the interface significantly depends on the viscosity $(0<\bar{h}<30 \mathrm{~mm})$, the mean value of the first cumulant decays relatively rapidly; the values of $\left\langle\Gamma_{1}\right\rangle$ are stabilized during passage to the exponential decay mode of $\bar{V}$ and decay during passage to the critical mode of interface pinning $(\bar{h}>50 \mathrm{~mm})$.

As mentioned above, the dependence of $\tau_{\lambda}$ on the parameter that determines the mobility of the scattering centers is determined by the character of their motion on a microscopic level. Analysis of the growth dynamics of local instabilities of the phase interface under noncoherent illumination (see Figs. 4 and 6) points to a predominantly diffusive character of microscopic motion of the liquid phase in the growing local instabilities of the interface in the critical mode (as $f \longrightarrow 0$ ). The parameter $S_{i, \text { aval }} \propto \xi_{i \text {, aval }}^{2}\left(\xi_{i \text {, aval }}\right.$ is the characteristic size of the $i$ th instability) is a linear function of time, at least at the initial stage of the growth of instabilities (see Fig. 6), which is typical of diffusive growth processes $\left\langle\xi_{i \text {, aval }}^{2}\right\rangle \propto D t$, where $D$ in this case is the effective diffusion coefficient of the liquid phase in a porous layer.

The decay of autocorrelation functions of the intensity fluctuations of speckles in the region of active growth of the interface (Fig. 7b), which admits an exponential approximation of the form $g_{2}(\tau)=\exp (-\Gamma|\tau|)$ with satisfactory accuracy, is also evidence in favor of the diffusive type of motion of the scattering centers as $f \longrightarrow 0$. Note that a nearly exponential decay of $g_{2}(\tau)$ with the increase in $\tau$ is indeed typical of single and small-angle multiple scattering of laser radiation in systems with diffusive motion of scattering centers. However, a substantial difference in the diffusion coefficients for different scattering centers should lead to a deviation in $g_{2}(\tau)$ from the canonical exponential form [23]. Therefore, the experimental behavior of $g_{2}(\tau)$ serves only as an indirect evidence of the diffusive character of motion of the liquid phase. At the same time, the results of analysis of images of local inhomogeneities under noncoherent illumination are indisputable evidence for the above conclusion.

Under the conditions of a speckle-correlation experiment, images form due to stochastic interference of multiply scattered components of incident radiation that propagate in the forward direction in a layer at small angles to the axis of incident radiation. When determining the coefficient $C_{C}$, which establishes a relation between the first cumulant $\Gamma_{1}$, determined in the speckle-correlation experiment, and the parameter $\tau_{\lambda}$, which characterizes the dynamics of scattering centers (local phase interfaces in pores), one should take 
into account both the average number $\langle n\rangle$ of acts of scattering of these components in the layer and the geometry of the experiment, which determines the characteristic value of the scattering angle $\varphi$ of the detected components (Fig. 10). Assuming that the scattering angle $\varphi_{i}$ in each act of scattering is a random variable with zero mean and considering the formation of each small-angle component as a sequence of statistically independent acts of scattering, we can estimate the rms angle of scattering for the components detected by the optical system as $\sigma_{\varphi_{\text {out }}} \approx \varphi_{\max } / 3 \sqrt{\langle n\rangle}$, where $\varphi_{\max }$ is the limit angle of detection (Fig. 10). A coefficient of $1 / 3$ is attributed to the application of the $3 \sigma$ rule to the sum of $\varphi_{i}$.

Since for a single small-angle scattering we have

$$
\Gamma_{1}=4 k^{2} \sin ^{2}(\varphi / 2) D \approx k^{2} \varphi^{2} D=\varphi^{2} / \tau_{\lambda},
$$

for the case of multiple small-angle scattering we can write

$$
\Gamma_{1} \approx\langle n\rangle \sigma_{\varphi_{\text {out }}}^{2} / \tau_{\lambda}=\varphi_{\max }^{2} / 9 \tau_{\lambda}
$$

the normalization factor $C_{C}$ for the model of formation of speckle-modulated images does not depend on $\langle n\rangle$ and is approximately $\varphi_{\max }^{2} / 9$. Using the geometrical parameters of the detection scheme (Fig. 10), we can approximately estimate $C_{C}$ as $2.22 \times 10^{-5}$.

Under the assumption of the isotropic character of the growth of local instabilities at the initial stage, we can estimate the coefficient $D$ within the model of twodimensional diffusion as $3.4 \times 10^{3} \mu^{2} / \mathrm{s}$ (for $f=0.07$ ). On the other hand, $\Gamma_{1} \approx 10.7 \pm 1.3 \mathrm{~s}^{-1}$ for $f=0.07$ (see Fig. 9). Using the value of $D$ from the data of Fig. 6, we determine $\tau_{\lambda} \approx 1.5 \times 10^{-6} \mathrm{~s}$ (for liquid-saturated paper, we have $n_{r} \approx 1.4$ [25]) and find that the coefficient $C_{C} \approx$ $(1.6 \pm 0.3) \times 10^{-5}$ estimated form the experimental data agrees satisfactorily with the model value (in spite of the assumptions made in calculating the latter). The estimates show the appropriateness of the scheme of the speckle-correlation experiment based on the detection of radiation scattered forward at small angles. Owing to the very small value of $C_{C}$, one can detect intensity fluctuations of speckles with a CCD camera with relatively small frame repetition.

Note that the value of $\left\langle\Gamma_{1}\right\rangle$ determined in passing from the Lucas-Washburn mode to the pinning stage is not as large as the average velocity $\bar{V}$ determined in experiments with noncoherent light (see Fig. 2). This is associated with the fact that the macroscopic mobility of the interface in the critical mode, which is characterized by $\bar{V}$, depends on both $\overline{\left\langle\partial S_{i, \text { aval }} / \partial t\right\rangle}$ and $\bar{N}_{\text {aval }}$ (formula (4)). The quantity $\left\langle\Gamma_{1}\right\rangle$ obtained from correlation analysis describes the effective value of the diffusion coefficient of a liquid in the layer: $\left\langle\Gamma_{1}\right\rangle \propto D \propto$ $\overline{\left\langle\partial S_{i, \text { aval }} / \partial t\right\rangle}$. A comparison of the critical indices $\gamma$ and $\delta$ and analysis of the dynamics of local instabilities as

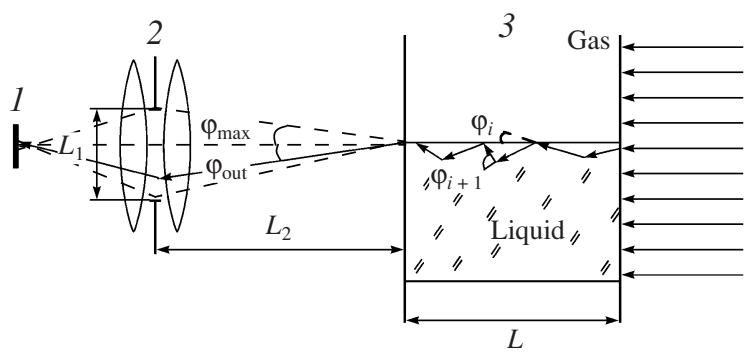

Fig. 10. Geometrical parameters of the detection scheme of scattered radiation in a speckle-correlation experiment; (1) CCD sensor, (2) objective lens, and (3) test sample. $L_{1}=$ $15 \mathrm{~mm}, L_{2}=530 \mathrm{~mm}, L \approx 100 \mu \mathrm{m}, \varphi_{\max } \approx L_{1} / 2 L_{2} \approx$ $0.014 \mathrm{rad}, \varphi_{\text {out }}=\Sigma \varphi_{i}$, and $\sigma_{\varphi_{\text {out }}}^{2}=\langle n\rangle \sigma_{\varphi_{i}}^{2}$.

$f \longrightarrow 0$ (see Fig. 6) show that the coefficient $D \propto$ $\overline{\left\langle\partial S_{i, \text { aval }} / \partial t\right\rangle}$ weakly depends on $f$ compared with the average velocity of the interface. Under the assumption of the scaling behavior of $\left\langle\Gamma_{1}\right\rangle$ (and, accordingly, under the assumption of an effective value of the diffusion coefficient of the liquid in the layer), analysis of the data shown in Fig. 9 for $f \longrightarrow 0\left(\left\langle\Gamma_{1}\right\rangle \propto D \sim f^{\delta^{\prime}}\right)$ allows us to evaluate the corresponding critical index as $\delta^{\prime} \approx$ $0.242 \pm 0.080$, which agrees satisfactorily with the value of the critical index $\delta \approx 0.19$ obtained from the analysis of the functions $\tilde{N}_{\text {aval }}(f)$ and $\bar{V}(f)$ (see Fig. 5).

\subsection{Critical Indices: Discussion of Experimental Results}

According to an approach considered by many authors (see, for example, $[10,11]$ ), the scaling properties of the interface between liquid and gaseous phases in a disordered porous medium with $f \longrightarrow 0$ can be described by a set of critical indices $z, v$, and $\alpha$. The dynamic critical index $z$ determines the relation between the characteristic size $L$ of a porous system and the characteristic time $t_{x}$ of growth of a phase interface: $t_{x} \propto L^{z}$. At the stage of interface pinning, for a growth time of the interface much greater than $t_{x}$, the width of the phase interface, defined as $W(L, t)=$ $\left\langle\sqrt{\overline{h^{2}(x, t)}-\overline{h(x, t)}}\right\rangle$, depends on the characteristic size of the system as $W_{\text {sat }} \sim L^{\alpha}$, where the critical index $\alpha$ describes the fractal properties of the developing interface. When determining the interface width, we used the following notation: $h(x, t)$ is a random height of the phase interface at point $x$ at instant $t$, the $x$ axis is perpendicular to the direction of motion of the interface, and the averaging is performed both over coordinate $x$ and over an ensemble of configurations of the phase interface that are randomly implemented at instant $t$. The critical index $v$ determines the scaling of the characteristic size $\xi$ of pinned regions of the interface (and, hence, the local instabilities of the interface) as $f \longrightarrow 0: \xi \propto f^{-v}$. The relation between $\xi$ and the char- 
acteristic time of the appearance and growth of a local instability of the interface is also determined by the dynamic critical index: $\tau_{\text {aval }} \propto \xi^{z}$ (see, for example, [20]). It should be noted $[10,11]$ that the characteristic longitudinal $\xi_{\|}=\xi$ (in the direction $x$ ) and transverse $\xi_{\perp}$ dimensions of local instabilities of the interface exhibit different scaling behavior: $\xi_{\perp} / \xi_{\|} \propto f^{v(1-\alpha)}$. Many authors (see, for example [10]) give a fundamental relation between the critical indices $z, v$, and $\alpha$ and the critical index of the average velocity of the interface at the stage of pinning: $\theta=v(z-\alpha)$.

It is interesting to analyze the relation of the critical indices $\gamma$ and $\delta$ with $z, v$, and $\alpha$ and to compare the experimental values with the data obtained by other authors. At the pinning stage as $f \longrightarrow 0$, we represent $\overline{\left\langle\partial S_{i, \text { aval }}(t) / \partial t\right\rangle}$ as $\overline{\left\langle\partial S_{i, \text { aval }}(t) / \partial t\right\rangle} \sim f^{\delta} \sim \xi_{\|} \xi_{\perp} / \tau_{\text {aval }}$. Taking into account the above scaling relations for $\xi_{\|}$, $\xi_{\perp}$, and $\tau_{\text {aval }}$, we find that $\delta=v(z-1-\alpha)$. Taking into account that $\theta=\gamma+\delta=v(z-\alpha)$, we obtain $\gamma=v$; i.e., $\bar{N}_{\text {aval }} \sim f^{\gamma} \sim 1 / \xi_{\|}$.

In $[10,11]$, the authors have discussed the critical exponents obtained from numerical simulation of the motion of phase interfaces in disordered media with the different models (in particular, models of directed percolation corresponding to the Kardar-Parisi-Zhang equation [26] and quenched Edwards-Wilkinson models corresponding to the Edwards-Wilkinson equation [27]). Note that the critical index $\theta$ for two-dimensional directed percolation depinning (DPD) models, which ranges from 0.47 to 0.82 (depending on the simulation error), agrees much better with our results than the appropriate values for quenched Edwards-Wilkinson models, which range from 0.21 to 0.33 .

The weak dependence of $\overline{\left\langle\partial S_{i \text {, aval }}(t) / \partial t\right\rangle}$ on the critical parameter, which was established by interpreting experimental data, is attributed to the factor $z-1-$ $\alpha$ in the expression given above, which establishes a relation between the critical indices $\delta$ and $z, \alpha$. Note that, in [10], which was devoted to analysis of the scaling properties of the interface between liquid and gaseous phases by numerical simulation on the basis of DPD models and full-scale experiments (capillary rise of a liquid in paper sheets), the authors obtained the following value of the critical index: $\alpha \approx 0.63$. The dynamic critical index $z$ for critical systems similar to those considered here is usually assumed to be about $14 / 9[11,20]$. For this value of the dynamic critical index, the value of $z-1-\alpha$ is close to zero, although the authors of [10] give smaller values of $z$ (in particular, for two-dimensional systems, simulation yields a value of 1.01). On the other hand, the authors of [20], using an experimental value of $\theta>1$, suggest that the values of the dynamic critical index are greater than 14/9.

The value of $\gamma \approx 0.57$, which we obtained for an FM sample, is much smaller than the critical index $v=v_{\|}$ determined by numerical simulation on the basis of a two-dimensional DPD model $[10,11]\left(v_{\|} \approx 1.73\right)$. On the other hand, analysis of the Kardar-Parisi-Zhang equation by the renorm group method [28] yields the following relation between $v$ and $\alpha: v=1 /(2-\alpha)$. Thus, for $\alpha \approx 0.63 \mathrm{~mm}$, we obtain $v \approx 0.73$, which agrees satisfactorily with the value of $\gamma$ obtained in our study. Note also that $v$ strongly depends on the dimension of the system: when $\xi_{\|}, \xi_{\perp} \leq H$, where $H$ is the layer thickness, we should take into account the three-dimensional character of the motion of the interface in a layer; according to the results of simulation in [10], this should lead to a decrease in $v_{\|}$and $v_{\perp}$ and to an increase in $z$. Anyway, the problem of the relationship between $\gamma$ and $v$, as well as the problem of scaling the average growth rate of local instabilities of the interface as $f \longrightarrow 0$, requires further experimental and theoretical investigation.

\section{CONCLUSIONS}

Thus, by experimentally investigating the critical behavior of the interface between liquid and gaseous phases in disordered porous layers, we have established that the critical parameter $f$ has a small effect on the growth dynamics of local instabilities of the interface (avalanches). At the pinning stage, the growth of the interface is mainly controlled by the dependence of the appearance rate of local instabilities on the critical parameter. The weak dependence of the average growth rate of local instabilities on $f$, established in experiments, is presumably associated with the small value of the difference between the dynamic critical index $z$ and the term $1+\alpha$, which depends on the structural properties of the growing interface (in particular, on its fractal dimension). On a microscopic level, this is associated with relatively weak decay of the effective diffusion coefficient of a liquid in a porous medium with the decrease of the critical parameter. The characteristic critical index of the average velocity in the disordered porous samples considered in the present paper is smaller than unity; this corresponds to a finite time of the interface pinning process (in contrast to porous media consisting of densely packed monodisperse spherical particles, which are characterized by a higher degree of structural order). Our results are of interest for describing the transport properties of porous systems of different natures in the case of the critical mode of liquid transfer in such objects.

\section{ACKNOWLEDGMENTS}

This work was supported by the Russian Foundation for Basic Research, project no. 07-02-01467a.

\section{REFERENCES}

1. S. Bhattacharya, M. J. Higgins, and J. P. Stokes, Phys. Rev. Lett. 63, 1503 (1989). 
2. P.-Z. Wong and J. W. Cable, Phys. Rev. B: Condens. Matter 28, 5361 (1983).

3. J. P. Stokes, M. J. Higgins, A. P. Kushnick, S. Bhattacharya, and M. O. Robbins, Phys. Rev. Lett. 65, 1885 (1990).

4. J. Koplik and H. Levine, Phys. Rev. B: Condens. Matter 32, 280 (1985).

5. T. Natterman, S. Stepanow, L.-H. Tang, and H. Leschhorn, J. Phys. II 2, 1483 (1992).

6. O. Narayan and D. S. Fisher, Phys. Rev. B: Condens. Matter 48, 7030 (1993).

7. D. Yu. Ivanov, Critical Behavior of Non-Ideal Systems (Fizmatlit, Moscow, 2003; Wiley, Weinheim, 2008), Section 1.2.

8. S. V. Buldyrev, A.-L. Barabási, F. Caserta, S. Havlin, H. E. Stanley, and T. Vicsek, Phys. Rev. A: At., Mol., Opt. Phys. 45, R8313 (1992).

9. L.-H. Tang and H. Leschhorn, Phys. Rev. A: At., Mol., Opt. Phys. 45, R8309 (1992).

10. L. A. N. Amaral, A.-L. Barabási, S. V. Buldyrev, S. T. Harrington, S. Havlin, R. Sadr-Lahijany, and H. E. Stanley, Phys. Rev. E: Stat. Phys., Plasmas, Fluids, Relat. Interdiscip. Top. 51, 4655 (1995).

11. L. A. N. Amaral, A.-L. Barabási, H. A. Makse, and H. E. Stanley, Phys. Rev. E: Stat. Phys., Plasmas, Fluids, Relat. Interdiscip. Top. 52, 4087 (1995).

12. T. H. Kwon, A. E. Hopkins, and S. E. O'Donnell, Phys. Rev. E: Stat. Phys., Plasmas, Fluids, Relat. Interdiscip. Top. 54, 685 (1996).

13. D. A. Zimnyakov, P. V. Zakharov, V. A. Trifonov, and O. I. Chanilov, Pis'ma Zh. Éksp. Teor. Fiz. 74 (4), 237 (2001) [JETP Lett. 74 (4), 216 (2001)].

14. D. A. Zimnyakov, A. P. Sviridov, L. V. Kuznetsova, S. A. Baranov, and N. Yu. Ignatieva, Appl. Opt. 45, 4480 (2006).
15. R. Otnes and L. Enochson, Applied Time Series Analysis (Wiley, New York, 1978; Mir, Moscow, 1982).

16. E. W. Washburn, Phys. Rev. 17, 273 (1921).

17. D. J. Durian, D. A. Weitz, and D. J. Pine, Science (Washington) 52, 686 (1991).

18. D. J. Durian, D. A. Weitz, and D. J. Pine, Phys. Rev. A: At., Mol., Opt. Phys. 44, R7902 (1991).

19. A. Marmur and R. D. Cohen, J. Colloid Interface Sci. 189, 299 (1997).

20. T. Delker, D. B. Pengra, and P. Z. Wong, Phys. Rev. Lett. 76, 2902 (1996)

21. M. Alava and K. Niskanen, Rep. Prog. Phys. 69, 669 (2006).

22. O. Zic, T. Kustanovich, E. Moses, and Z. Olami, Phys. Rev. E: Stat. Phys., Plasmas, Fluids, Relat. Interdiscip. Top. 58, 689 (1998).

23. Photon Correlation and Light Bearing Spectroscopy, Ed. by H. Cummins and E. Pike (Plenum, New York, 1974; Mir, Moscow, 1978).

24. A. Ishimaru, Wave Propagation and Scattering Media (Academic, New York, 1978, Mir, Moscow, 1981), Vol. 2.

25. D. A. Zimnyakov, L. V. Kuznetsova, O. V. Ushakova, and R. Myullyulya, Kvantovaya Élektron. (Moscow) 37, 9 (2007).

26. Z. Csahók, K. Honda, and T. Vicsek, J. Phys. A: Math. Gen. 26, L171 (1993).

27. R. Bruinsma and G. Aeppli, Phys. Rev. Lett. 52, 1547 (1984).

28. G. Grinstein and S. K. Ma, Phys. Rev. B: Condens. Matter 28, 2588 (1983).

Translated by I. Nikitin 